\title{
Predicting the Outcome of Nerve Conduction Studies in Patients with Suspected Carpal Tunnel Syndrome: Using an Existing Carpal Tunnel Assessment Tool
}

\author{
Carl Edwards ${ }^{1,2^{*}}$, Ian Frampton ${ }^{2}$ \\ ${ }^{1}$ Torbay Hospital, Torquay, UK \\ ${ }^{2}$ University of Exeter, Exeter, UK \\ Email: ${ }^{*}$ carl.edwards@nhs.net
}

Received 7 February 2014; revised 20 March 2014; accepted 1 April 2014

Copyright (C) 2014 by authors and Scientific Research Publishing Inc.

This work is licensed under the Creative Commons Attribution International License (CC BY). http://creativecommons.org/licenses/by/4.0/

c) (i) Open Access

\begin{abstract}
This service evaluation and pilot study was designed to establish whether a clinical questionnaire could be incorporated within our Secondary Care Carpal Tunnel Service. The purpose of the questionnaire is to predict the positive and negative results of Nerve Conduction Studies (NCS) in those patients with suspected Carpal Tunnel Syndrome. The hand specialist, preceding NCS administered the questionnaire; it was then scored at a later date. Results showed a sensitivity of $86 \%$ and specificity of $84 \%$ referring to the ability to predict a positive NCS when using a predetermined cut-off score. When analysed with Receiver Operating Characteristics, a threshold score could be determined in order to obtain $100 \%$ sensitivity/specificity. This questionnaire can be used as a useful adjunct to assessment of those presenting with suspected Carpal Tunnel Syndrome. Using the questionnaire to identify those patients scoring outside a predetermined threshold range would reduce the need for NCS by nearly $\mathbf{5 0 \%}$, with significant cost and clinical practice implications.
\end{abstract}

\section{Keywords}

Carpal Tunnel Syndrome, Questionnaire, Nerve Conduction Studies, Health Economics

\section{Introduction}

Carpal tunnel syndrome (CTS) is the most common type of peripheral nerve entrapment [1]. Symptoms can be

${ }^{*}$ Corresponding author.

How to cite this paper: Edwards, C. and Frampton, I. (2014) Predicting the Outcome of Nerve Conduction Studies in Patients with Suspected Carpal Tunnel Syndrome: Using an Existing Carpal Tunnel Assessment Tool. Open Journal of Therapy and Rehabilitation, 2, 57-62. http://dx.doi.org/10.4236/ojtr.2014.22010 
debilitating including loss of sensation in the fingers, pain, muscle wasting, weakness of grip and night waking. The condition is most commonly seen in females and in the age range 40 - 50 [2]. The pathology involves the compression of the median nerve passing through the carpal tunnel in the hand. The tunnel is exactly that, with the "floor and walls" composed of the carpus and the "roof" by the flexor retinaculum, a fibrous ligamentous structure attaching to the pisiform and hamate medially and scaphoid and trapezium laterally. Through the carpal tunnel, the tendons of flexor digitorum profundus, flexor digitorum superficialis, flexor pollucis longus and the median nerve pass [2].

Numerous studies have attempted to link occupational history with the development of this condition. These have been generally proved inconclusive with those in heavy construction as likely to develop the condition as those within office-based employment. Conolly and McKessar [3] suggest that most cases of CTS are constitutional, although some patients in occupations involving increased force and pressure within the carpal tunnel have an increased risk of developing CTS. Mechanical changes affecting either the structure of the "tunnel", or its contents can affect the median nerve. For example, previous distal radius fractures with associated deformity, or flexor synovitis presenting commonly in inflammatory arthropathy.

CTS is by definition a collection of signs and symptoms that in combination make the clinical diagnosis relatively straightforward [4]. Clinical history and presentation are paramount in diagnosing this condition, however further investigative measures are available to help clarify the diagnosis and severity of compression.

Differential diagnosis is crucial. Paraesthesia in the hand is a common complaint and while it is easy to label a patient with CTS, other potential causes have to be considered. The median nerve originates from the C6/7 nerve roots, so any compression of the nerve or its roots could culminate in paraesthesia in the hand. More proximal compression of the median nerve may include the pronator teres or struthers arcade. Inflammatory conditions need to be considered such as mononeuritis and inflammatory demyelinising neuropathy. Radiculopathy, thoracic outlet, polyneuropathies are among other potential differential diagnoses thus stressing the importance of reaching a confident diagnosis before considering treatment [5].

If clinical diagnosis is in doubt, further investigative methods are available. The most widely researched and used are Nerve Conduction Studies (NCS), which are the best predictor of symptom severity [4]. Descatha et al. [6] showed that NCS together with clinical examination significantly improved detection rates for CTS compared to examination alone. They suggested that clinical examination is appropriate as an initial screening, and that NCS should be used as the confirmatory test in those who have less convincing signs and symptoms on examination. Although NCS are often considered to be the gold standard, they are open to both operator and interpreting error. Nevertheless, it does provide a useful adjunct to clinical assessment in localising potential nerve entrapments and assisting with surgical decision-making [7].

Questionnaires have been developed to help predict the likelihood of CTS [8]-[10]. These have typically included diagrams, which are annotated by the patient reflecting the distribution of their symptoms together with multiple choice and single answer questions. An assessment tool developed by Kamath and Stothard [9] based on work carried out by Levine et al. [8] and further studies by Bridges et al. [11] has proven to be a reliable adjunct to developing a diagnosis. It comprises a list of questions to be used within the assessment process by the clinician. Responses are scored using a simple algorithm with weighted scores for each answer. These have been based on six critical domains that were identified by a panel of hand surgeons, rheumatologists and patients [8]. These domains include pain, paraesthesia, numbness, weakness, nocturnal symptoms and over-all functional status and used to predict likelihood of CTS. Previous studies [9] have shown that this assessment tool has a sensitivity of $85 \%$ in predicting CTS compared with the result of subsequent surgery.

Since CTS can be diagnosed with this kind of systematic assessment, we wondered whether it is possible to predict whether or not a patient is so likely to be "classical" for CTS that NCS could be acceptably removed from the assessment process. If it is possible to identify those who do not need NCS in order to make a positive or negative diagnosis (because they have so many or so few of the "classic" symptoms respectively), there could be significant savings in clinic time, increased capacity and reduced costs.

The aim of the current study is therefore to explore the development and preliminary evaluation of a paperbased questionnaire based on Kamath and Stothard [9] in a clinical series of patients referred for assessment and diagnosis of CTS. The objectives of the study are to derive upper and lower threshold scores to predict positive and negative diagnosis of CTS and to compare these with current clinical diagnosis including examination and NCS. 


\section{Method}

\subsection{Participants}

68 patients attending a local secondary care clinic for assessment of CTS consented to participate in the study. Exclusion criteria included any possible peripheral neuropathy (for example-those with diabetes mellitus, since diagnosis of CTS in patients with diabetic neuropathy is difficult as the two conditions may affect the median nerve in a similar way [12] [13]). Renal transplant patients (often requiring surgery including the removal of amyloid tissue) and pregnant patients were also excluded.

\subsection{Materials}

Participants were asked a series of standard questions (see Appendix) by the first author at the beginning of their appointment. Clinical assessment was then conducted as normal in order to make a clinical diagnosis of CTS. Tests included assessment of sensation, autonomic function, tinel, phalens and flick test. Cervical spine range was assessed and any resultant exacerbation of distal symptoms was recorded.

NCS was based on the Kamath and Stothard [9] study. Criteria for normal values were matched, with terminal latency to APB less than $4.0 \mathrm{~ms}$ and a sensory conduction from digit 2 to wrist greater than $47 \mathrm{~m} / \mathrm{s}$. Further routine tests included transpalmar recording to digit 3 with a $20 \%$ reduction in conduction velocity for the median nerve across the carpal tunnel compared to the palm to finger recording considered significant.

Following the appointment the questionnaires were scored and compared to the results of the NCS and clinical diagnosis.

\subsection{Data Analysis}

Questionnaire data were scored using the algorithm developed by Kamath and Stothard [9]. Cut-off thresholds for predicting positive and negative NCS were derived using Receiver Operating Characteristics (ROC) analyses [14].

\section{Results}

The questionnaire was completed on 68 patients, of whom 50 (73\%) tested positive for CTS on NCS, 19 (27\%) tested negative. Figure 1 depicts the frequencies of questionnaire total scores for NCS-positive and NCS-negative diagnosis.

ROC analyses showed that of those testing NCS-positive 41 (82\%) scored five or over on the questionnaire. Of those testing NCS-negative 15 (83.3\%) scored below five on the questionnaire. All those scoring 7 or above went on to have a positive NCS, and those scoring 1 or less all presented with negative NCS.

$20 \%$ of respondents $(n=14)$ indicated that they had previously received a carpal tunnel injection. All of these had had good, temporary, symptomatic relief of symptoms, and all proved to have positive NCS and Questionnaire scores.

Using the original cut off score as 5 or above as a positive questionnaire result [9] we can compare to the results of NCS using these two discrete dichotomous variables.

\begin{tabular}{ccc} 
& Score 5 or Over & Score under 5 \\
\hline Positive NCS & 41 & 9 \\
Negative NCS & 3 & 15 \\
\hline
\end{tabular}

McNemar Change Tests: Pearson chi2: 3 ( $\mathrm{p}=0.0833$ ); Yates chi2: 2.083; $(\mathrm{p}=0.1489)$; Binomial $(\mathrm{p} \geq 3 \mid \mathrm{N}=12)=0.9807$.

\section{Discussion}

The aim of the present study was to explore the potential for a standardised questionnaire to predict outcome of nerve conduction studies (NCS) for patients referred for diagnosis of potential carpal tunnel syndrome (CTS). 68 participants attending a clinical diagnostic service for the assessment of CTS completed a brief standard clinician-administered questionnaire before undergoing routine clinical assessment and diagnosis. Using Receiver Operating Characteristic (ROC) analyses, a single threshold score was derived to predict the outcome of NCS 


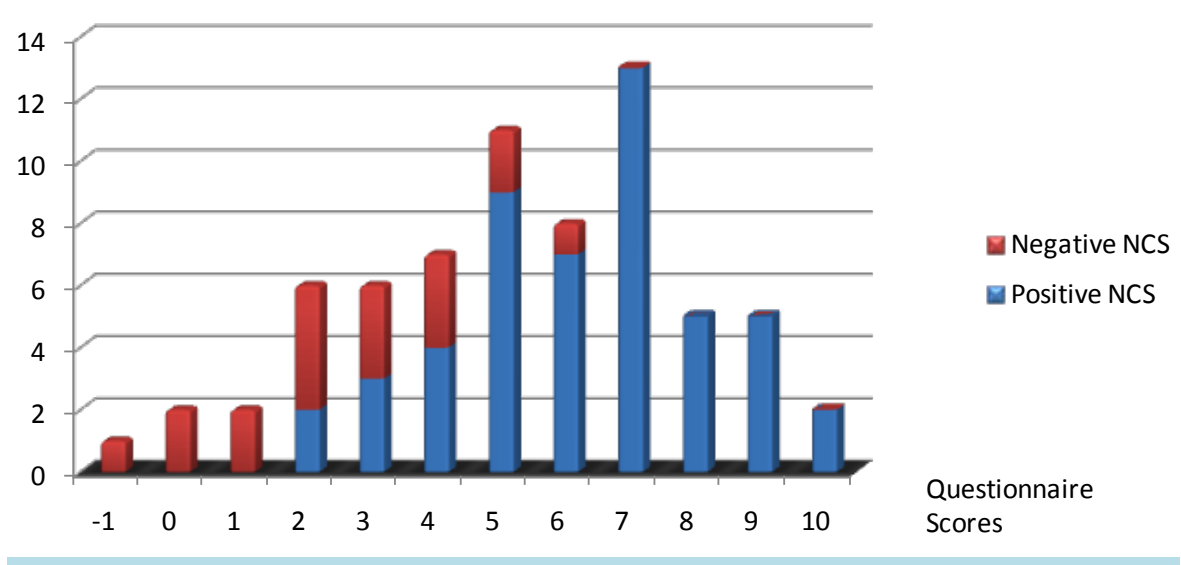

Figure 1. Questionnaire score by NCS positive and negative diagnosis.

with a sensitivity of $82 \%$ (i.e. 16 out of 20 patients with positive NCS were correctly identified by those scoring five or more on the questionnaire) and a specificity of $83.3 \%$ (i.e. 17 out of 20 patients with a negative NCS were correctly identified by those scoring less than five on the questionnaire).

Adopting this single cut-off in the present sample led to $17 \%$ false positives and $18 \%$ false negatives. Nine patients scored below the questionnaire threshold yet went on to have positive NCS results, suggesting that a single cut-off threshold may not be sensitive enough in clinical practice. Correlation between NCS and questionnaire scores based upon the single cut-off was assessed using McNemar Chi-squared testing. The results do not show a significant correlation, however it is important to bear in mind the contents of the $2 \times 2$ table used to evaluate this statistic. Relating once more to the clinical context, the important category is that of the patients which present having scored over 5 yet have negative NCS $(n=3)$, it could be hypothesised that these patients may well be listed for surgery having bypassed NCS. It must be remembered however that CTS can still be present despite there being normal NCS. Those patients who presented with positive NCS yet scored below five $(n=9)$ would be clinically "safe" as they would have proceeded to have NCS carried out as a result of their low scoring questionnaire and therefore "captured".

One way of improving the results would be to devise threshold range, which could capture both the false negatives and positives. Looking specifically at the results of the present study all those scoring seven or over went on to have positive NCS and those scoring one or below all had negative tests.

Using this approach it would be reasonable to consider offering those scoring between 2 - 6 further confirmatory NCS and those outside of that range a shorter appointment without NCS (sensitivity and specificity 100\%). $56 \%$ of the patients $(n=38)$ fell within the threshold range of $2-6$ and on this basis would require additional NCS tests in order to make a clinical diagnosis. The remaining $44 \%(n=30)$ were outside of the threshold and on this basis would not require NCS in order to make a clinical diagnosis.

The potential impact, if this model was used, could be very significant. Based on a notional annual referral rate of 600 patients with $44 \%$ not requiring studies this could save 66 hours of clinic time amounting to 19 whole orthopaedic clinics. This would provide more flexibility of the service, either to see more patients with CTS or to expand other existing services.

Patient expectation factors should also be considered. Patients often attend clinic with a clear expectation as to what they want to gain from their appointment. From their discussion with their family doctor or referring consultant a suspected diagnosis has often been discussed. With access to the Internet and other resources readily available patients may well look into the condition and the expected presentation, thus influencing their answers.

In order to justify any potential change in a service, the effect on both patient satisfaction and their journey through the system needs to be evaluated. Although not looked at within this study it would be reasonable to assume that a patient would rather wait to have the correct diagnosis rather than opt for a rapid assessment service. A study carried out by Khu et al. [1] looking at patients' perceptions of carpal tunnel surgery and ulnar nerve decompression surgery found that satisfaction was clearly linked with clinical outcome, which in turn was dependant on the correct initial diagnosis. With this in mind rapid access to treatment may not be the only relevant factor.

Plans have been made to explore this further through a subsequent study. This will explore the potential of 
using this tool as a self-completed questionnaire as a method of screening for nerve conduction studies in primary care before making an onward referral. Together with this item analysis and logistic regression will be carried out in order to maximise the potential of this useful assessment tool. The statistical analysis should optimise the algorithm to provide the most reliable results.

\section{Conclusion}

The results from this study demonstrate the potential for incorporating a standardised clinical questionnaire in the assessment of CTS. Further investigation of the psychometric properties of the questionnaire would help to establish whether it could be used in a self-completed format at the primary care stage to help referrers identify those patients who require further NCS in order to make an accurate diagnosis, and those who can reliably refer on for a clinical diagnosis and treatment without further investigation. Potential savings could be made within both primary care (where inappropriate referral may be avoided), and secondary care (reducing number of onward referrals for NCS) together with benefits to patients reducing potential lengthy waits without compromising the quality of their journey.

\section{Acknowledgements}

Dr Ian Frampton and Dr Karen Knapp Department of Psychology, University of Exeter; Mrs Frances Hunt and Mrs Roberta Ainsworth Physiotherapy Department Torbay Hospital for their help and guidance and time.

\section{References}

[1] Khu, K.J., Bernstein, M. and Midha, R. (2011) Patients Perception of Carpal Tunnel and Ulnar Nerve Decompression Surgery. Canadian Journal of Neurological Sciences, 38, 268-273.

[2] Luchetti, R. and Amadio, P. (2007) Carpal Tunnel Syndrome. Springer, Berlin. http://dx.doi.org/10.1007/978-3-540-49008-1

[3] Conolly, W.B. and McKessar, J.H. (2009) Carpal Tunnel Syndrome—Can It Be a Work Related Condition? Australian Family Physician, 38, 684-686.

[4] Patijn, J., Vallejo, R., Janssen, M., Huygen, F., Lataster, A., van Kleef, M. and Mekhail, N. (2011) Carpal Tunnel Syndrome. Pain Practice, 11, 297-301. http://dx.doi.org/10.1111/j.1533-2500.2011.00457.x

[5] Petiot, P. and Bernard, E. (2011) Diagnostic Pitfalls in Carpal Tunnel Syndrome. Revue Neurologique (Paris), 167, 64-71. http://dx.doi.org/10.1016/j.neurol.2010.08.009

[6] Descatha, A., Dale, A.M., Franzblau, A., Coomes, J. and Evanoff, B. (2010) Diagnostic Strategies Using Physical Examination Are Minimally Useful in Defining Carpal Tunnel Syndrome in Population-Based Research Studies. Occupational and Environmental Medicine, 67, 133-135. http://dx.doi.org/10.1136/oem.2009.047431

[7] Strickland, J.W. and Gozani, S.N. (2011) Accuracy of In-Office Nerve Conduction Studies for Median Nerve: A Meta-Analysis. Journal of Hand Surgery, 36, 52-60. http://dx.doi.org/10.1016/j.jhsa.2010.09.012

[8] Levine, D.W., Simmons, B.P., Koris, M.J., Daltroy, L.H., Hohl, G.G., Fossel, A.H. and Katz, J.N. (1993) A Self-Administered Questionnaire for the Assessment of Symptoms and Functional Status in Carpal Tunnel Syndrome. The Journal of Bone and Joint Surgery, 75, 1585-1592.

[9] Kamath, V. and Stothard, J. (2003) A Clinical Questionnaire for the Diagnosis of Carpal Tunnel Syndrome. The Journal of Hand Surgery, 28, 455-459. http://dx.doi.org/10.1016/S0266-7681(03)00151-7

[10] Katz, J.N., Stirrat, C.R., Larson, M.G., Fossel, A.H., Eaton, H.M. and Liang, M.H. (1990) A Self-Administered Hand Symptom Diagram for the Diagnosis and Epidemiologic Study of Carpal Tunnel Syndrome. Journal of Rheumatology, 17, 1495-1498.

[11] Bridges, M.J., Robertson, D.C. and Chuck, A.J. (2011) Predicting the Result of Nerve Conduction Studies in Carpal Tunnel Syndrome Using a Questionnaire. Hand Surgery, 16, 39-42. http://dx.doi.org/10.1142/S0218810411005047

[12] Bahrmann, A., Zieschang, T., Neumann, T., Hein, G. and Oster, P. (2010) Carpal Tunnel Syndrome in Diabetes Mellitus. Medizinische Klinik, 105, 150-154. ( in German) http://dx.doi.org/10.1007/s00063-010-1024-8

[13] Gazioglu, S., Boz, C. and Cakmak, V.A. (2011) Electrodiagnosis of Carpal Tunnel Syndrome in Patients with Diabetic Polyneuropathy. Clinical Neurophysiology, 122, 1463-1469. http://dx.doi.org/10.1016/j.clinph.2010.11.021

[14] Fombonne, E. and Fuhrer, R. (1992) Use of ROC Analysis in Psychiatry. Encephale, 18, 545-550. (in French) 


\section{Appendix}

\section{Carpal Tunnel Questionnaire [9]}

HISTORY (circle yes/no number)

Has pain in the wrist woken you up at night?

Yes $1 \quad$ No 0

Has tingling and numbness in your hand woken you during the night?

Yes $1 \quad$ No 0

Has tingling and numbness in your hand been more pronounced first thing in the morning?

Yes $1 \quad$ No 0

Do you have any trick movements to make the tingling, numbness go from your hands?

Yes $1 \quad$ No 0

Do you have any tingling or numbness in your little finger at any time?

Yes $0 \quad$ No 3

Has tingling and numbness presented when you were reading a newspaper, steering a car or knitting?

Yes 1 No 0

Do you have any neck pain?

Yes $-1 \quad$ No 0

If applicable has the tingling and numbness in your hand been severe during pregnancy?

Yes $1 \quad$ No $-1 \quad$ N/A 0

Has it helped the tingling and numbness on wearing a splint on your wrist?

Yes 2 No $0 \quad$ N/A 0 